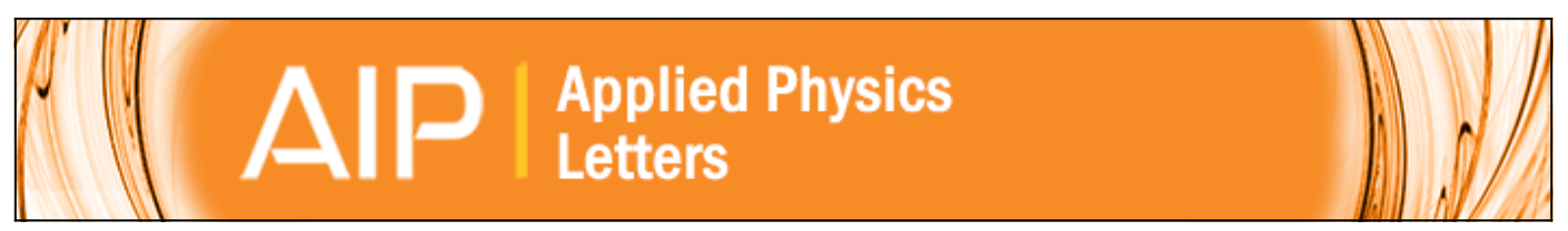

Enhanced broad-band extraordinary optical transmission through subwavelength perforated metallic films on strongly polarizable substrates

Tianyi Sun, Eser Metin Akinoglu, Chuanfei Guo, Trilochan Paudel, Jinwei Gao, Yang Wang, Michael Giersig, Zhifeng Ren, and Krzysztof Kempa

Citation: Applied Physics Letters 102, 101114 (2013); doi: 10.1063/1.4795151

View online: http://dx.doi.org/10.1063/1.4795151

View Table of Contents: http://scitation.aip.org/content/aip/journal/apl/102/10?ver=pdfcov

Published by the AIP Publishing 


\title{
Enhanced broad-band extraordinary optical transmission through subwavelength perforated metallic films on strongly polarizable substrates
}

\author{
Tianyi Sun, ${ }^{1,2, a)}$ Eser Metin Akinoglu, ${ }^{3, a)}$ Chuanfei Guo, ${ }^{2}$ Trilochan Paudel, ${ }^{1}$ Jinwei Gao, ${ }^{4}$ \\ Yang Wang, ${ }^{4}$ Michael Giersig, ${ }^{3}$ Zhifeng Ren, ${ }^{2}$ and Krzysztof Kempa ${ }^{1, b)}$ \\ ${ }^{1}$ Department of Physics, Boston College, Chestnut Hill, Massachusetts 02467, USA \\ ${ }^{2}$ Department of Physics and TcSUH, University of Houston, Houston, Texas 77204 \\ ${ }^{3}$ Department of Physics, Freie Universität Berlin, Berlin, Germany \\ ${ }^{4}$ South China Normal University, Guangzhou 510006, People's Republic of China
}

(Received 14 January 2013; accepted 27 February 2013; published online 15 March 2013)

\begin{abstract}
We demonstrate through simulations and experiments that a perforated metallic film, with subwavelength perforation dimensions and spacing, deposited on a substrate with a sufficiently large dielectric constant, can develop a broad-band frequency window where the transmittance of light into the substrate becomes essentially equal to that in the film absence. We show that the location of this broad-band extraordinary optical transmission window can be engineered in a wide frequency range (from IR to UV), by varying the geometry and the material of the perforated film as well as the dielectric constant of the substrate. This effect could be useful in the development of transparent conducting electrodes for various photonic and photovoltaic devices. @ 2013 American Institute of Physics. [http://dx.doi.org/10.1063/1.4795151]
\end{abstract}

It has been demonstrated that a subwavelength perforated metallic film (SPMF) can, surprisingly, not only transmit light but can do it very efficiently. ${ }^{1-5}$ This extraordinary optical transmittance (EOT) is extraordinary for two reasons. First, EOT occurs even when the perforation dimensions are much smaller than the light wavelength, which seemingly violates conventional wave optics. ${ }^{6}$ Second, the fraction of light transmitted exceeds fraction of surface area covered by perforation. This indicates that light falling on the metallic surface in-between perforations is channeled through neighboring perforations. EOT can be mediated by the surface plasmon polaritons (SPP) coupled to the external radiation via grating effect (Umklapp). ${ }^{1}$ However, it was demonstrated that this mechanism cannot explain EOT in some cases of perforated films with complex perforation geometries. ${ }^{7}$ Instead, in this case, SPMF can be considered as an effective plasmonic medium, which consists of an array of parallel waveguides (perforations), and its effective dielectric function has (in the simplest model) the plasma Drude form $\varepsilon_{f} \sim 1-\omega_{p}{ }^{2} / \omega^{2}$, with the plasma frequency $\omega_{p} \approx 2 c / a \sqrt{\varepsilon_{b}}$, where $c$ is the speed of light in vacuum, $a$ is the perforation size, and $\varepsilon_{b}$ is the dielectric constant of the background. ${ }^{8,9} \mathrm{In}$ this effective medium picture, EOT is the induced transparency of the "plasma" above the effective plasma frequency $\omega_{p}$. In another effective medium approach, the dielectric function of SPMF can be quantitatively estimated by first considering its Babinet complementary pattern (an array of isolated metallic islands) and then transforming it back to the perforation array. ${ }^{10,11}$ It was shown that this method is equivalent to the parallel waveguide approach. ${ }^{8,9,12}$ Here, we demonstrate an aspect of EOT not mentioned before: the SPMF deposited on a substrate with sufficiently large

\footnotetext{
a) T. Sun and E. M. Akinoglu contributed equally to this work.

${ }^{b)}$ Author to whom correspondence should be addressed. Electronic mail: kempa@bc.edu.
}

permittivity develops a broad-band frequency window, where the transmittance of light into the substrate becomes essentially equal to that without the film.

A simple model captures the main features of this effect and illustrates the importance of large dielectric constant of the substrate. Consider first a planar dielectric film with thickness $t_{f} \ll \lambda$ (wavelength in vacuum) and the dielectric constant $\varepsilon_{f}$, deposited at a planar interface between vacuum and an infinitely thick substrate with the dielectric constant $\varepsilon_{s u b}$ (see Fig. 1(a)). For simplicity, we assume here that all dielectrics are lossless, and that all electromagnetic waves propagate perpendicular to the interfaces. The relative change in transmittance into the substrate due to the presence of the film is ${ }^{13-15}$

$$
\tau=\frac{T}{T_{0}}=\frac{1}{1-\alpha},
$$

where $T$ and $T_{0}$ are the transmittances into the substrate with and without the dielectric film, respectively (see Fig. 1(a)), and

$$
\alpha \approx \frac{4\left(1-\varepsilon_{f}\right)\left(\varepsilon_{f}-\varepsilon_{\text {sub }}\right)}{\left(1+\sqrt{\varepsilon_{s u b}}\right)^{2}}\left(\frac{t_{f} \omega}{c}\right)^{2}
$$

Note that, if

$$
1<\varepsilon_{f}<\varepsilon_{s u b}
$$

$\alpha$ is positive and thus $\tau$ is greater than 1 . This implies $T>T_{0}$, i.e., that the presence of the film actually improves the transparency. This is the well-known effect of the dielectric constant grading (DCG) in dielectric film stacks, employed often to improve wave propagation through interfaces between media with large dielectric constant contrast. Here, $T>T_{0}$ implies also $R<R_{0}$, and so DCG acts somewhat similar to the anti-reflection coating (ARC). However, in contrast to ARC, it is not a result of a destructive interference, 
(a)

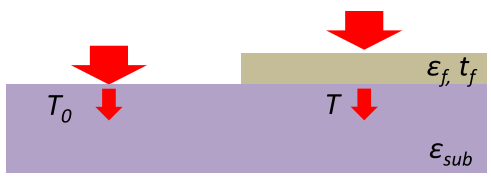

(b)

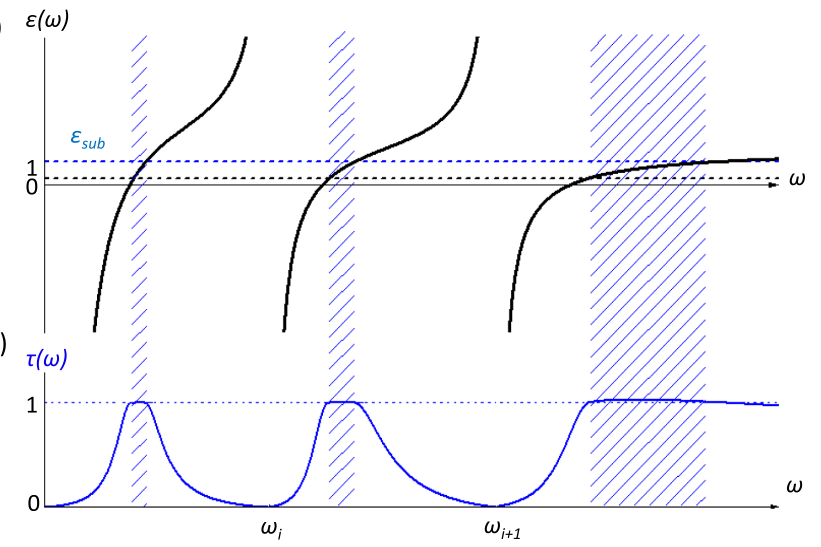

FIG. 1. (a) Schematic of the model structures (b) Sketch of a typical effective dielectric function $\varepsilon_{f}$ versus frequency, for a typical (lossless) SPMF model, given by Eq. (4). (c) Sketch of the normalized transmittance $\tau$ versus frequency, for a SPMF model with dielectric losses included. The shaded regions represent the condition (3) and thus approximate locations of the EOT windows. Note that the "broadest" EOT window occurs for $\omega>\omega_{N}$.

which normally requires $t_{f}$ to be of order $\lambda$, to match the phase. Note that unless $\varepsilon_{f}$ is singular, $\alpha$ which is of the order of $\left(t_{f} / \lambda\right)^{2}$ is small, and thus $\tau \approx 1$. To continue with model calculations, we now assume that SPMF can be treated as the simple dielectric film of Fig. 1(a), but with a frequency dependent $\varepsilon_{f}$. This indeed is possible ${ }^{10,16}$ and for lossless SPMF this effective dielectric function is

$$
\varepsilon_{f}(\omega)=\varepsilon_{b}+\sum_{i=1}^{N} \frac{A_{i}}{\omega_{i}^{2}-\omega^{2}},
$$

where $\varepsilon_{b}$ is the background dielectric constant, $\omega_{i}$ are the plasmonic resonant frequencies, and $A_{i}$ are the oscillator strengths. The resonance with highest frequency occurs for $\omega=\omega_{N}$. A sketch of $\varepsilon_{f}$ vs $\omega$ is shown in Fig. 1(b). In contrast to the case of a simple dielectric, here $\varepsilon_{f}$ diverges near the poles (plasmonic resonances) and consequently so does $\alpha$ (given by Eq. (2)). As a result, $\tau$ is expected to approach zero at the poles but can be large in-between the resonances in the EOT windows, approximately given by the DCG condition (Eq. (3)), and shown as the shaded areas in Figs. 1(b) and 1(c).

Fig. 1(c) shows a sketch of $\tau$ for this model structure, but with losses included. This can be accomplished by simply allowing the dielectric constants to become complex in the analysis above. In this case, Eq. (4) must be modified by replacing $\omega^{2}$ with $\omega(\omega+i \gamma)$, where $\gamma$ is the loss factor. The resulting equations lose their simplicity, but the main effect of the losses is simply to smooth-out the singularities and reduce $\tau$. Also, even though Eq. (3) is no longer valid, it can still be used (with real parts of $\varepsilon_{f}$ and $\varepsilon_{s u b}$ ) as a rough guide of the EOT window location; note that the shaded areas coincide with the maxima of $\tau$ in Fig. 1(c). Fig. 1(c) confirms qualitatively all conclusions, which can be drawn from Fig. 1(b). In particular it is clear that the EOT windows have different widths, but that the widest is the one for frequencies higher than $\omega_{N}$ (highest plasmonic resonance). Thus, if maximum width of the EOT window is desired, all plasmonic resonances must be engineered to occur below the required frequency window.

This engineering can be achieved by changing the film geometry (e.g., perforation shapes and dimensions, period, thickness) and material (e.g., $\mathrm{Ag}, \mathrm{Au}, \mathrm{Al}$ ), as well as by choosing the dielectric environment of the SPMF (e.g., $\varepsilon_{\text {sub }}$ ). Of particular importance is the effect of $\varepsilon_{\text {sub }}$. First, it is clear from the condition (3) that $\varepsilon_{s u b}$ directly controls the width of the EOT window, which linearly increases with $\varepsilon_{\text {sub }}$. Second, increasing $\varepsilon_{\text {sub }}$ reduces the frequencies of all plasmonic resonances, since it increases the dielectric constant of the environment $\varepsilon_{e n v}$ of the electrons in SPMF, and all the resonance frequencies scale as $1 / \sqrt{\varepsilon_{e n v}}$.

To verify the above conclusions, we have developed a series of samples containing various SPMF structures, involving different metals as well as transparent and opaque substrates. Subsequently, a series of experiments of optical transmittance have been performed, as well as corresponding simulations, based on the finite difference time domain (FDTD) method. The SPMFs were made by applying the nanosphere lithography. ${ }^{17,18}$ Polystyrene (PS) latex spheres (dispersed in water) with submicron diameters were dispersed in an ethanol-water solution and slowly applied to a water surface in a Petri dish using a glass pipet. A selfassembly of the spheres on the water surface formed a hexagonal closed-packed, monolayer with very low defect density, which was subsequently deposited onto clean substrates by evaporation of the water. Subsequently, the spheres were attached to the substrate surfaces, and their diameters had been reduced by means of reactive ion etching $\left(100 \% \mathrm{O}_{2}\right)$. Afterwards, the obtained structure served as a mask for deposition of metallic films. The metallic films were deposited on top of a $2 \mathrm{~nm}$ thick Ti buffer layer by means of electron-beam evaporation at a base pressure of $10^{-7}$ millibars. Subsequently, PS spheres were removed by thermal and chemical treatment. The last step of the preparation included the cleaning and rinsing of the surface with propanol and drying under $\mathrm{Ar}$ flow. In the FDTD simulation, ${ }^{19-21}$ metallic films with hexagonal hole arrays of different radii were positioned on semi-infinite substrates. Power fluxes were monitored at the SPMF/substrate interface to determine the transmittance into the substrate at each wavelength. Perfectly matched layer boundary conditions were used in the vertical direction to prevent unphysical scattering at the edge of the simulation unit cell. Periodic boundary conditions were applied to in-plane dimensions of the unit cell to simulate the infinite large films. Published experimental data were used for all dielectric functions. ${ }^{22,23}$

The results for all these samples confirm the conclusions above, and we show here selected experimental-simulation results, which demonstrate this. The first result shown is for a structure with SPMF with hexagonal array of circular perforations ( $a=460 \mathrm{~nm}$ diameter, the spacing between holes $l=756 \mathrm{~nm}$ ) made in $50 \mathrm{~nm}$ thick gold film on a sapphire substrate $\left(\varepsilon_{\text {sub }}=3\right)$. The transmittance of sapphire and the gold SPMF on it were recorded from far field measurements in Perkin Elmer photo-spectrometer with an incident angle of $0^{\circ}$ in respect to the normal of the surface. The measured, 
(a)
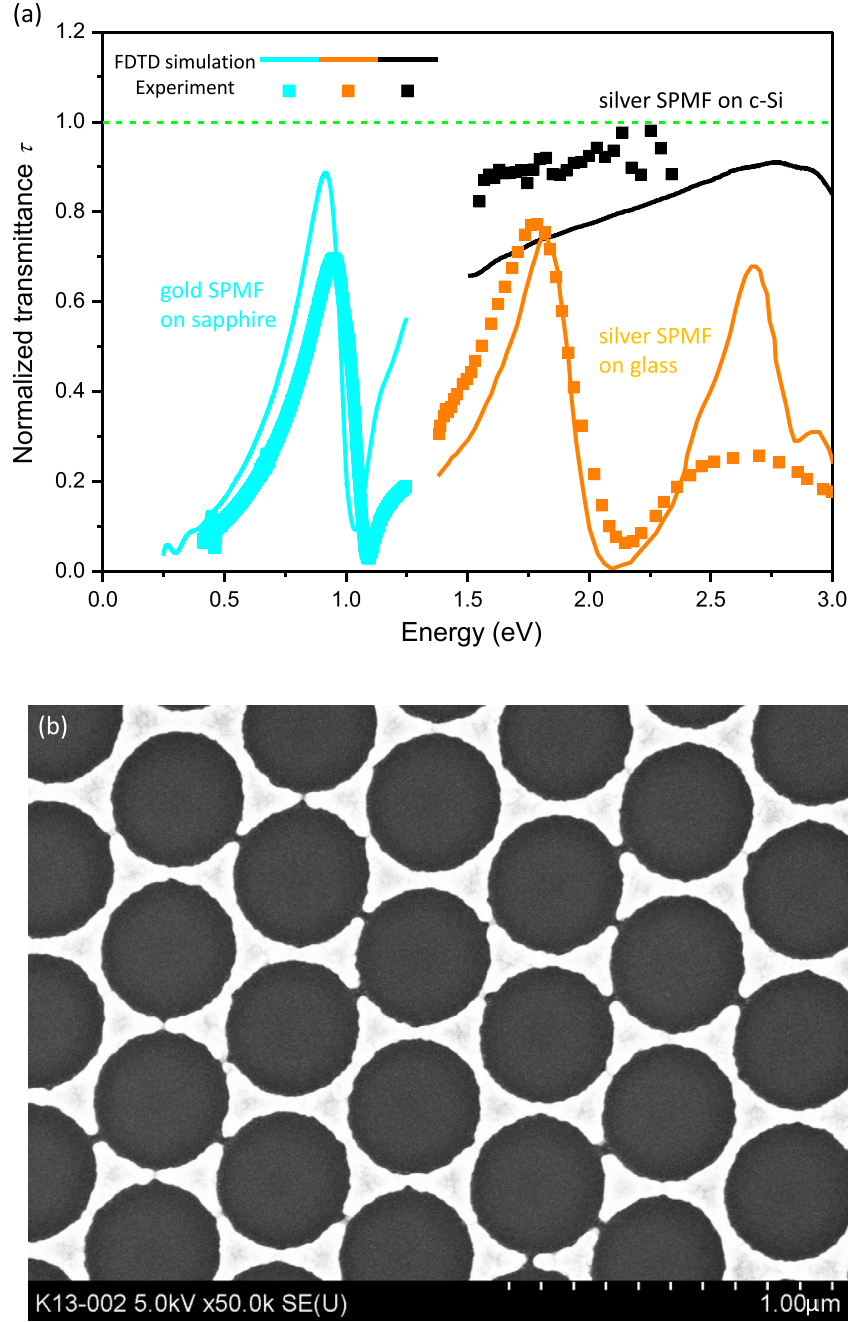

FIG. 2. (a) FDTD simulated (solid curves) and measured (solid squares) normalized transmittance $\tau$ of three SPMFs, each with a hexagonal array of holes: color cyan- $\left(a=460 \mathrm{~nm}, l=756 \mathrm{~nm}, t_{f}=50 \mathrm{~nm}\right)$ gold film on a sapphire substrate; color orange- $\left(a=196 \mathrm{~nm}, l=400 \mathrm{~nm}, t_{f}=50 \mathrm{~nm}\right)$ silver film on a glass substrate (taken from Ref. 24); color black( $a=420 \mathrm{~nm}, l=470 \mathrm{~nm}, t_{f}=50 \mathrm{~nm}$ ) silver film on a $c$-Si substrate. (b) SEM image of the silver SPMF on an ultra-thin $c$-Si substrate.

normalized transmittance $\tau$ for this structure is shown by cyan-solid squares in Fig. 2(a). The corresponding FDTD simulation is represented by the cyan-solid curve and is in excellent agreement with the experiment. The pronounced peak at $0.9 \mathrm{eV}$ corresponds to the first EOT window in the vicinity of the longitudinal plasmon (where $\varepsilon_{f}$ is vanishing). The deep minimum at $1.1 \mathrm{eV}$, on the other hand, represents the trapped plasmonic resonance at $\omega_{1}$. To show the universality of the transmittance properties (including the lineshape), we present in Fig. 2(a) also the result taken from the Ref. 24, for SPMF with similar hexagonal array of circular perforations in $50 \mathrm{~nm}$ silver film on glass $\left(\varepsilon_{\text {sub }}=2.3\right)$, with similar spacing between holes $(l=400 \mathrm{~nm})$, but with very small perforations ( $a=196 \mathrm{~nm}$ ). The strongly reduced diameter of the perforations (holes) leads to increased frequency of all resonances, which scale roughly as $1 / a$. In particular, the $\omega_{1}$ plasmonic resonance (minimum of $\tau$ ) occurs now at $\sim 2.2 \mathrm{eV}$, i.e., approximately by a factor of $\sim 2$ higher, than the resonance for the gold SPMF (cyan-solid squares and line), in agreement with the expected scaling. The experimental result is again in agreement with the FDTD simulation. Since this is the first EOT window, it is necessarily narrow (comparable to the gold SPMF case), as expected.

To engineer a much broader EOT window, we have designed a SPMF structure, again with a hexagonal array of circular perforations in $50 \mathrm{~nm}$ silver film, with a $=420 \mathrm{~nm}$ and $l=470 \mathrm{~nm}$, but deposited on a crystalline silicon $(c-\mathrm{Si})$ substrate, which has a very large dielectric constant $\left(\varepsilon_{\text {sub }} \approx 16\right)$. FDTD simulations for this structure, shown in Fig. 2(a) as black-solid curve, confirm that in this case the EOT window is very broad (with $\tau>0.7$ in $1.5 \mathrm{eV}<\hbar \omega<$ $3 \mathrm{eV}$ ). This is expected, since with the very large $\varepsilon_{\text {sub }}$, all plasmonic resonances are strongly renormalized below 1.5 $\mathrm{eV}$, and thus the range shown represents the last (and thus very broad) EOT window for $\omega>\omega_{N}$.

To demonstrate this very broad EOT window experimentally, an ultra-thin substrate is required to allow for a direct measurement of the transmittance. Accordingly, we have deposited SPMF with the designed geometry (as in the simulations above) on an ultra-thin $c$-Si substrate, of only $5 \mu \mathrm{m}$ thickness (p-type $c$-Si wafer, double side polished to true mirror quality, University Wafer). The SEM image of this structure is shown in Fig. 2(b). Such an ultra-thin $c$-Si substrate remains transparent in a large frequency range of the visible spectrum, allowing the direct measurements. The transmittance of the ultra-thin silicon and SPMF on it was measured with the EQE system QE-PV-Si, Oriel, Newport, USA. The results are shown in Fig. 2(a) as the black-solid squares. In spite of a relatively large scatter of the data at high frequencies, due to the reduced transmission signal through the substrate, the agreement with the simulations is good and demonstrates the presence of the very broad EOT window in the expected frequency range. In fact, the measured EOT window is even better than the simulated one, with $\tau>0.9$ in the entire measured range $(1.5 \mathrm{eV}<\omega<2.25 \mathrm{eV})$.

This demonstrated possibility of engineering the EOT window is of practical importance. For instance, it could help in the development of transparent conducting electrodes for photonics and photovoltaics. In fact, the unusual good performance of the recently developed solar cell with SPMF, similar to ours, ${ }^{25}$ could be related or even directly caused by the large dielectric constant of the substrate and similar mechanism. Finally, our simulations (not shown here) demonstrate that this effect can be further enhanced (increased $\tau$ and the bandwidth of the EOT window) by combining SPMF with the conventional ARC, made of an optically thick dielectric film.

In conclusion, we study theoretically and experimentally transmittance through SPMF structures with various geometries and material combinations. We demonstrate with calculations, simulations, and experiments that an enhanced, broad-band EOT window can be engineered for a SPMF, by structure engineering and by employing substrates with large dielectric constant. This enhanced broad-band EOT can be further enhanced by combining with the conventional ARC and could be used to develop transparent and electrically conducting electrodes for photonics and photovoltaics.

The work performed at Boston College and University of Houston is funded by the U.S. Department of Energy under Contract No. DOE DEFG02- 00ER45805 (Z.F.R.). 
The work performed at SCNU was supported by the Guangdong Leadership Award, and the National Science Foundation of China. M.G. acknowledges a financial support from Helmholtz-Zentrum Berlin for Materials and Energy $\mathrm{GmbH}$.

${ }^{1}$ T. W. Ebbesen, H. J. Leek, H. F. Ghaemi, T. Thio, and P. A. Wolff, Nature 391, 667-669 (1998).

${ }^{2}$ H. J. Lezec, A. Degiron, E. Devaux, R. A. Linke, L. Martin-Moreno, F. J. Garcia-Vidal, and T. W. Ebbesen, Science 297, 820-822 (2002).

${ }^{3}$ L. Martin-Moreno, F. J. Garcia-Vidal, H. J. Lezec, K. M Pellerin, T. Thio, J. B. Pendry, and T. W. Ebbesen, Phys. Rev. Lett. 86, 1114-1117 (2001).

${ }^{4}$ K. J. Klein Koerkamp, S. Enoch, F. B. Segerink, N. F. van Hulst, and L. Kuipers, Phys. Rev. Lett. 92, 183901 (2004).

${ }^{5}$ H. Liu and P. Lalanne, Nature 452, 728-731 (2008).

${ }^{6}$ H. Bethe, Phys. Rev. 66, 163-182 (1944).

${ }^{7}$ W.-C. Chen, N. I. Landy, K. Kempa, and W. J. Padilla, "A subwavelength extraordinary-optical-transmission channel in Babinet metamaterials," Adv. Opt. Mat. (published online).

${ }^{8}$ J. B. Pendry, L. Martn-Moreno, and F. J. Garcia-Vidal, Science 305, 847-848 (2004).

${ }^{9}$ F. J. Garcia-Vidal, L. Martn-Moreno, and J. B. Pendry, J. Opt. A: Pure Appl. Opt. 7, S97-S101 (2005).

${ }^{10}$ K. Kempa, Phys. Status Solidi (RRL) 4, 218-220 (2010).
${ }^{11}$ Y. Peng, T. Paudel, W. Chen, W. J. Padilla, Z. F. Ren, and K. Kempa, Appl. Phys. Lett. 97, 041901 (2010).

${ }^{12}$ Y. Wang, E. W. Plummer, and K. Kempa, Adv. Phys. 60, 799-898 (2011).

${ }^{13}$ J. D. Jackson, Classical Electrodynamics, 3rd ed. (Wiley, New York, 1998).

${ }^{14} \mathrm{~J}$. A. Kong, Electromagnetic Wave Theory (EMW, Cambridge, MA, 2005).

${ }^{15}$ O. S. Heavens, Optical Properties of Thin Solid Films (Dover, New York, 1965).

${ }^{16}$ K. Kempa, Phys. Rev. B 74, 033411 (2006).

${ }^{17}$ A. Kosiorek, W. Kandulski, P. Chudzinski, K. Kempa, and M. Giersig, Nano Lett. 4, 1359 (2004).

${ }^{18}$ G. Ctistis, P. Patoka, X. Wang, K. Kempa, and M. Giersig, Nano Lett. 7, 2926-2930 (2007).

${ }^{19}$ A. Taflove and S. C. Hagness, Computational Electrodynamics: The Finite-Difference Time-Domain Method (Artech, Norwood, MA, 1995).

${ }^{20}$ MIT Electromagnetic Equation Propagation, Massachusetts Institute of Technology (http://ab-initio.mit.edu/wiki/index.php/Meep)

${ }^{21}$ CST Microwave Studio, the Computer Simulation Technology AG (http:// www.cst.com/Content/Products/MWS/Overview.aspx)

${ }^{22}$ D. Palik, Handbook of Optical Constants of Solids (Academic, Boston, 1985).

${ }^{23}$ P. B. Johnson and R. W. Christy, Phys. Rev. B 6, 4370 (1972).

${ }^{24}$ S. H. Lee, K. C. Bantz, N. C. Lindquist, S. Oh, and C. L. Haynes, Langmuir 25(23), 13685-13693 (2009).

${ }^{25}$ J. Zhu, X. Zhu, R. Hoekstra, L. Li, F. Xiu, M. Xue, B. Zeng, and K. L. Wang, Appl. Phys. Lett. 100, 143109 (2012). 